\title{
Testosterone deficiency caused by castration increases adiposity in male rats in a tissue-specific and diet-dependent manner
}

Myunggi Baik ${ }^{1,2,3^{*}+} \mathbb{D}$, Jin Young Jeong ${ }^{4 \dagger}$, Seung Ju Park ${ }^{1,2}$, Seon Pil Yoo ${ }^{1,2}$, Jin Oh. Lee ${ }^{1,2}$, Jae Sung Lee ${ }^{1,2}$, Md Najmul Haque ${ }^{1,2}$ and Hyun-Jeong Lee ${ }^{4}$

\begin{abstract}
Background: Testosterone deficiency in men is clinically associated with the development of metabolic syndrome, which manifests as obesity, hepatic steatosis, and type-2 diabetes. We investigated the effects of castration-induced testosterone deficiency on body adiposity and the expression of genes related to lipid metabolism and glucose uptake and androgen signaling in male rats fed a normal diet (ND) or a high-fat diet (HFD).

Methods: Changes in lipid and glucose metabolism and androgen signaling were investigated at physiological and molecular levels in the muscle, liver, and adipose tissues of non-castrated and castrated rats under ND or HFD feeding.

Results: Castration-induced testosterone deficiency predisposed animals on ND to early development of fatty liver by activating fatty acid (FA) synthesis, whereas HFD activated hepatic FA uptake CD36 expression, leading to the development of hepatic steatosis. In rats fed ND, castration induced muscle fat accumulation by activating CD36 expression. In the subcutaneous fat of ND-fed rats, castration increased adiposity and the expression of FA synthesis-related genes, but it decreased glucose transporter gene expression. In the abdominal fat of rats fed ND, castration increased adiposity by upregulating FA synthesis-related genes, and HFD promoted adiposity by inducing FA uptake, glucose transporter, and FA synthesis-related gene expression. In rats fed ND, castration decreased body growth and muscle weight and downregulated the expression of genes androgen signaling in the longissimus dorsi muscle.
\end{abstract}

Conclusions: Testosterone deficiency increases adiposity in a tissue-specific and diet-dependent manner. Testosterone deficiency decreases body and muscle weights and downregulates androgen signaling.

Keywords: Castration, Testosterone deficiency, High-fat diet, Adiposity, Lipid and glucose metabolism

\footnotetext{
* Correspondence: mgbaik@snu.ac.kr

${ }^{\dagger}$ Myunggi Baik and Jin Young Jeong contributed equally to this work.

'Department of Agricultural Biotechnology, College of Agriculture and Life

Sciences, Seoul National University, 1 Gwanak-ro, Gwanak-gu, Seoul 08826,

Republic of Korea

${ }^{2}$ Research Institute of Agriculture and Life Sciences, College of Agriculture

and Life Sciences, Seoul National University, Seoul, Republic of Korea

Full list of author information is available at the end of the article
}

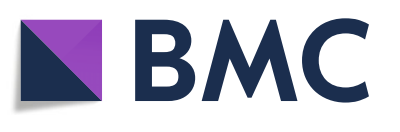

() The Author(s). 2020 Open Access This article is licensed under a Creative Commons Attribution 4.0 International License, which permits use, sharing, adaptation, distribution and reproduction in any medium or format, as long as you give

appropriate credit to the original author(s) and the source, provide a link to the Creative Commons licence, and indicate if changes were made. The images or other third party material in this article are included in the article's Creative Commons licence, unless indicated otherwise in a credit line to the material. If material is not included in the article's Creative Commons licence and your intended use is not permitted by statutory regulation or exceeds the permitted use, you will need to obtain permission directly from the copyright holder. To view a copy of this licence, visit http://creativecommons.org/licenses/by/4.0/. 


\section{Introduction}

Testosterone plays an important role in lipid and glucose metabolism [1]. Kelly et al. [2] suggested that testosterone reduces the deposition of body fat by regulating the expression of genes related to lipid and glucose metabolism. A low testosterone level or testosterone deficiency deregulates lipid and glucose metabolism, resulting in increased adiposity in the liver and peripheral tissues [2-5]. Testosterone deficiency is associated with increased visceral adiposity [6]. A low testosterone level is predictive of the development of metabolic syndrome, which manifests as obesity, nonalcoholic fatty liver disease, and type- 2 diabetes in men [1, 7] and laboratory animals [2, 5]. The effects of testosterone deficiency on adiposity and lipid/ glucose metabolism are controversial. For example, androgen deficiency in combination with a high-fat diet (HFD) exacerbated adiposity and insulin resistance in male mice [5]. In contrast, the serum glucose and insulin levels in male rats were unaffected by testosterone deficiency and an HFD [8]. Therefore, the effects of testosterone deficiency on lipid and glucose metabolism require further investigation.

The deposition of body fat is modulated by the lipid metabolic pathways that mediate fatty acid (FA) uptake, synthesis, and degradation [9-11]. The FA transporter CD36 mediates FA uptake and plays an important role in hepatic steatosis [12]. Limited information is available on the role of CD36 in fat deposition in testosteronedeficient rats. Kelly et al. [2] reported differences in the effect of testosterone on the function of subcutaneous (sc) versus visceral adipose tissue: testicular-feminized mice (with a non-functional androgen receptor [AR] and low testosterone level) exhibited reduced GLUT4 expression in sc, but not visceral adipose tissue. Further studies of the effects of testosterone deficiency on the expression of genes related to lipid and glucose metabolism in peripheral tissues (including the liver and skeletal muscle) and fat depots (sc and abdominal [ab] adipose tissues) are needed to understand the mechanisms by which adiposity is regulated.

Testosterone deficiency decreases growth and muscle mass in orchidectomy and AR-knockout mice [5] and reduces muscle mass in male humans [13]. These findings suggest the importance of testosterone for body and muscle growth. Testosterone signaling is mediated by the AR signaling pathway $[14,15]$, and thus investigation of the effect of testosterone deficiency on AR signaling is required.

Feeding an HFD to rodents alters their lipid and glucose metabolism [16, 17] and is often used to study metabolic syndrome such as visceral adiposity, hyperlipidemia, and insulin resistance [18]. The effect of the combination of testosterone deficiency and an HFD thus needs to be clarified. We hypothesized that testosterone deficiency differentially deregulates the expression of genes related to lipid metabolism and glucose uptake in a tissue-specific manner, predisposing to metabolic syndrome. We also hypothesized that an HFD exacerbates the testosterone deficiency-induced alterations in lipid and glucose metabolism. To test these hypotheses, we examined the effect of castration on the growth, adiposity, skeletal muscle mass, blood parameters, and expression levels of genes related to lipid metabolism and glucose uptake in the liver, muscle, and sc and ab fat depots in male rats fed a normal diet (ND) or an HFD for 9 weeks.

\section{Materials and methods}

\section{Animals, castration, and diets}

All experimental procedures involving animals were approved by the Chonnam National University (CNU) Institutional Animal Use and Care Committee (permission number CNU IACUC-YB-R-2010-13). All animal management procedures followed the standard operating protocols of CNU. Male Sprague-Dawley rats were purchased from Orient Bio (Gyeonggi-Do, Republic of Korea) and were maintained in a temperature (22 \pm $1{ }^{\circ} \mathrm{C}$ ) - and humidity (45-65\%)-controlled room under a $12 / 12 \mathrm{~h}$ light/dark cycle with ad libitum access to food and water.

Rats were castrated at 6 weeks of age, at which time their average body weight was $213 \mathrm{~g}$. All surgical instruments were pre-sterilized by acceptable methods, including steam sterilization. Male rats were anesthetized by intramuscular injection of Zoletil ${ }^{\mathrm{TM}} 50$ (5 mg/kg bodyweight, Virbac, France) and placed on a heating pad. The scrotum of the rats was shaved and cleaned using $70 \%$ ethanol. A small midline incision $(1 \mathrm{~cm})$ was made through the skin of the scrotum, and the testes were located, gently squeezed out, and removed. Next, the vas deferens, fat, and blood vessels were restored to their original position in the scrotal sac, and the vas deferens and blood vessels were ligated using silk sutures (Ailee, Seoul, Korea). The muscle layer and skin were closed with sutures, after which the rats were returned to their cages and their recovery monitored. The sham surgery control rats underwent an identical procedure as castrated rats except no removal of the testes.

After acclimatization for 1 week following castration with ND feeding, the rats were divided into the following four groups (8 per group): sham-operated control rats fed an ND or HFD and castrated rats fed an ND or HFD. The normal AIN93-G diet (D10012G) and HFD (D12451) were purchased from Research Diets, Inc. (New Brunswick, NJ, USA); their compositions are shown in Supporting Tables S1-S3. Briefly, in the HFD versus ND, $45 \%$ versus $16 \%$ of energy was from fat, $35 \%$ versus $64 \%$ from carbohydrates, and $20 \%$ versus $20 \%$ 
from protein, respectively. The food intake of the rats was recorded daily at the same time of day, and their body weight was measured weekly.

\section{Blood and tissue collection}

At the end of the 9-week feeding period, the rats (16 weeks of age) were fasted for $8 \mathrm{~h}$. The rats were anesthetized with $\mathrm{CO}_{2}$, and blood samples were collected via cardiac puncture, transferred into an ET tube without anticoagulant, and stored at $4{ }^{\circ} \mathrm{C}$. Blood samples were centrifuged at $2000 \times g$ for $20 \mathrm{~min}$ at $4{ }^{\circ} \mathrm{C}$ to collect serum, which was stored at $-80^{\circ} \mathrm{C}$ until analysis. After anesthetizing with $\mathrm{CO}_{2}$, the rats were euthanized by decapitation, and the liver, adipose tissues ( $\mathrm{ab}$ and $\mathrm{sc}$ ), and skeletal muscle tissues (longissimus dorsi, gastrocnemius, and soleus) were immediately removed and weighed. The tissues were frozen in liquid nitrogen and stored at $-80^{\circ} \mathrm{C}$ for subsequent determination of total lipid, mRNA, and protein levels.

\section{Serum and tissue analyses}

Serum parameters were analyzed as reported previously $[19,20]$. Briefly, the serum level of glucose was analyzed using hexokinase reagents (Green Cross Reference Laboratory, Seoul, Korea). The serum level of triglycerides was analyzed by enzymatic spectrophotometric assay (Green Cross Reference Laboratory). The serum levels of testosterone and insulin were analyzed by enzyme-linked immunosorbent assay. The serum level of free FAs was analyzed by the acyl-coenzyme A (CoA) synthase-acylCoA oxidase method. The serum levels of total cholesterol, high-density lipoprotein (HDL), and low-density lipoprotein (LDL) were analyzed by enzymatic colorimetric assays.

Muscle tissues were dissected and placed in liquid nitrogen. Total lipids were extracted from approximately $50 \mathrm{mg}$ muscle tissue by the Folch method [21].

\section{Histology}

For histological analysis, the liver was dissected and freed from any visible fat and blood. Histologic analysis and Oil red $\mathrm{O}(\mathrm{ORO})$ staining of tissues were performed as reported previously [20]. Briefly, liver specimens were fixed in $10 \%$ formalin, equilibrated in $20 \%$ sucrose, embedded in optimal cutting temperature compound, and cut into $7-\mu \mathrm{m}$-thick sections using a cryostat at $-20^{\circ} \mathrm{C}$. To evaluate fat deposition, frozen sections were fixed in $10 \%$ formalin, stained with $0.5 \% \mathrm{ORO}$, and examined under a microscope. The degree of liver ORO staining was determined and graded from 0 to 5 (lowest to highest).

\section{Real-time polymerase chain reaction}

Total RNA was isolated using TRIzol reagent (Molecular Research Center, Cincinnati, OH, USA) according to the manufacturer's instructions. Total RNA was quantified by measuring the absorbance at $260 \mathrm{~nm}$, and its integrity was assessed by agarose gel electrophoresis and ethidium bromide staining of the $28 \mathrm{~S}$ and 18S RNA bands. cDNA synthesis and real-time polymerase chain reaction analyses were performed as described previously [19]. The primers were designed using Integrated DNA Technology (Coralville, IA, USA) and the Primer 3 software based on sequences in GenBank and were synthesized by Bioneer (Daejeon, Korea). Information on the primers is shown in Supporting Table S4. The $2^{-\Delta \Delta C T}$ method was used to determine relative fold changes in gene expression levels normalized to the appropriate housekeeping gene (glyceraldehyde-3-phosphate dehydrogenase for liver, $\beta$-actin for muscle, and acidic ribosomal phosphoprotein $\mathrm{P0}$ for adipose tissue).

\section{Western blotting}

Western blotting was performed as described previously with slight modifications [20]. Briefly, samples were homogenized in protein extraction solution using a Polytron homogenizer (Thomas Scientific, Swedesboro, NJ, USA). The protein concentration of the samples was determined using the Bicinchoninic Acid Protein Assay Kit (Pierce, Rockford, IL, USA). Proteins were resolved by sodium dodecyl sulfate-polyacrylamide gel electrophoresis (SDS-PAGE) on either 6\% (for analysis of v-Akt murine thymoma viral oncogene homolog 1 [Akt] and phospho [p]-Akt, $60 \mathrm{kDa}$ ) or 10\% (for analysis of insulin receptor substrate-1 [IRS-1] and p-IRS-1, $180 \mathrm{kDa}$ ) polyacrylamide gels. The resolved proteins were transferred onto polyvinylidene fluoride membranes, blocked with $1 \times$ phosphate-buffered saline $/ 0.1 \%$ Tween 20 containing $5 \%$ non-fat dried milk, and incubated with the appropriate primary antibodies against Akt, p-Akt, IRS-1, and pIRS-1 (1:500 dilution). The mouse polyclonal anti-Akt, mouse monoclonal anti-p-Akt (Thr308), mouse polyclonal anti-IRS-1, and mouse monoclonal anti-p-IRS-1 antibodies were purchased from Cell Signaling Technologies (Danvers, MA, USA). Next, the blots were incubated in the presence of horseradish peroxidaseconjugated anti-mouse secondary antibodies (1:3000 dilution) (Santa Cruz Biotechnology, Santa Cruz, CA, USA) and developed using an enhanced chemiluminescence system (Thermo Scientific, Rockford, IL, USA). The processed blots were exposed to X-ray film, and the autoradiograms were analyzed using an HP scanner and Alpha Ease FC software. Band densities were normalized to that of the respective $\beta$ tubulin band. 


\section{Statistical analyses}

Data are presented as means \pm standard error (SE). Data were analyzed using the general linear model procedure in SAS (SAS Institute Inc., Cary, NC, USA). The model included the effects of castration, diet (ND or HFD), and the castration $\times$ diet interaction. When interactions were significant, the PDIFF option of LSMEANS in SAS was used to perform individual comparisons of the means. Differences were considered significant when the $P$ value was less than 0.05 .

\section{Results}

\section{Castration decreased body and muscle weight but} increased body adiposity

Castrated rats in the ND and HFD groups had a lower $(P<0.05)$ body weight from 5 to 9 weeks of feeding than that of rats in the sham-control group (Fig. 1a). Castration decreased $(P<0.05)$ the food intake of the rats, and the castrated rats showed a lower $(P<0.05)$ feed efficiency than that of the control rats (Table 1).

Castration increased $(P<0.05)$ the sc, ab, and total $(\mathrm{sc}+\mathrm{ab})$ fat weights in the ND and HFD groups (Table 1$)$. The liver weight was lower $(P<0.05)$ in the HFD group compared with the control group. The decreased liver weight may be due to decreased body weights in castrated animals fed HFD in this study. However, castration increased $(P<0.05)$ hepatic lipid accumulation in the ND group (Fig. 1b); this increase was significantly exacerbated by the HFD ( $P$ $<0.05)$. Castration reduced $(P<0.05)$ the longissimus dorsi muscle weight in the ND and HFD groups and reduced $(P<0.05)$ the gastrocnemius muscle weight in the ND group (Table 1). Castration increased $(P<$ $0.05)$ the longissimus dorsi muscle lipid content in the ND group but not in the HFD group (Fig. 1c).

\section{Castration influenced the serum glucose, free FA, and cholesterol concentrations}

As expected, castration decreased $(P<0.001)$ the serum concentration of testosterone irrespective of the type of diet (Table 2). Castration increased $(P<$ $0.05)$ the serum concentration of free FAs in the ND group but not in the HFD group. Castration increased $(P<0.05)$ the serum concentrations of total cholesterol, LDL, HDL, and glucose in the ND group. Castration did not affect the serum concentration of insulin or triglycerides. HFD feeding decreased $(P<$ 0.001 ) the serum concentration of insulin but increased $(P<0.05)$ the serum concentrations of total cholesterol and LDL. HFD may impair glucose signaling via suppressing insulin secretion in $\beta$-cell, as suggested by previous study [22].
Castration and an HFD modulate the expression of genes related to lipid metabolism and glucose uptake

Castration modulated the expression of genes related to lipid metabolism in a tissue-specific manner. Castration upregulated $(P<0.05)$ the expression of CD36 in the liver and in sc and ab fat in the HFD group but not in the ND group, while it upregulated $(P<0.05)$ the expression of CD36 in the muscle in the ND group but not in the HFD group (Fig. 2a). In the liver, castration upregulated $(P<0.05)$ the expression of acetyl-coA carboxylase $(A C C)$ and fatty acid synthase $(F A S N)$ in the ND group. In contrast, castration upregulated $(P<0.05)$ the expression of $A C C$ and FASN in muscle in the HFD group (Fig. 2b). In sc and ab fat, castration upregulated $(P<0.05)$ the expression of $A C C$ and/or FASN in both groups.

Castration did not affect GLUT2 expression in the liver or GLUT4 expression in muscle in both diet groups (Fig. 2c). In contrast, castration downregulated $(P<0.05)$ the expression of GLUT4 in sc fat in both groups (3-10\% of the level in the control group). In ab fat, castration upregulated GLUT4 expression in the HFD group but not in the ND group. The HFD decreased $(P<0.05)$ glucose transporter expression in the liver but not in the other tissues evaluated. Castration decreased $(P<0.05)$ the expression of LDL receptor $(L D L r)$ in the liver and muscle in the ND group (Fig. 2d). In muscle tissues, castration decreased $A R$ expression in both groups (Fig. 2d) and signal transducer and activator of transcription $5 \mathrm{a}$ $(S T A T 5 a)$ expression in the ND group. The HFD decreased $(P<0.05)$ the expression of $A R$ in the muscle. Neither castration nor diet type affected STAT5a and $A R$ expression in either the liver or sc fat (data not shown). Neither castration nor diet type affected the protein level of IRS-1, p-IRS-1, Akt, or p-Akt in any of the muscle tissues (Supporting Fig. S1).

\section{Discussion}

Testosterone deficiency increases body adiposity and deregulates expression of genes related to lipid metabolism and glucose uptake in tissue-specific and diet-dependent manners

In this study, we found that castration-induced testosterone deficiency increased adiposity in the liver, muscle, and sc and ab fat in rats fed an ND and also increased adiposity in the liver and Sc and ab fat, but not in the muscle, in rats fed an HFD. Consistent with our results, a low testosterone level or testosterone deficiency reportedly increases adiposity in the liver and several peripheral tissues [2, 3, 5], and an HFD increases tissue adiposity $[4,5,18]$.

We examined the expression levels of genes involved in FA uptake and synthesis to evaluate the molecular mechanisms underlying the effects of testosterone 


\section{A Body weight}

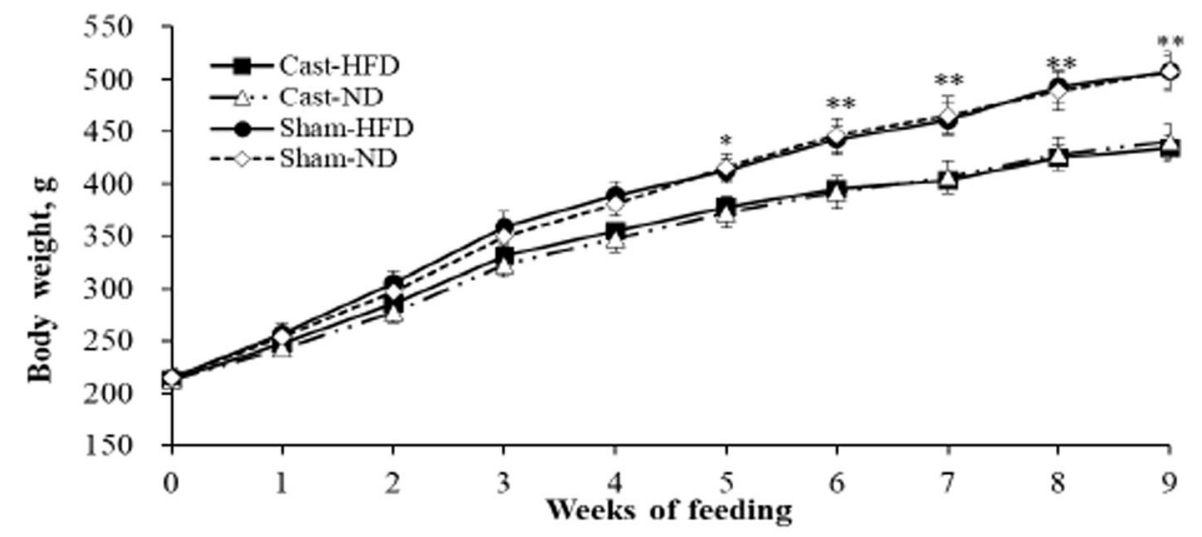

B Liver ORO staining
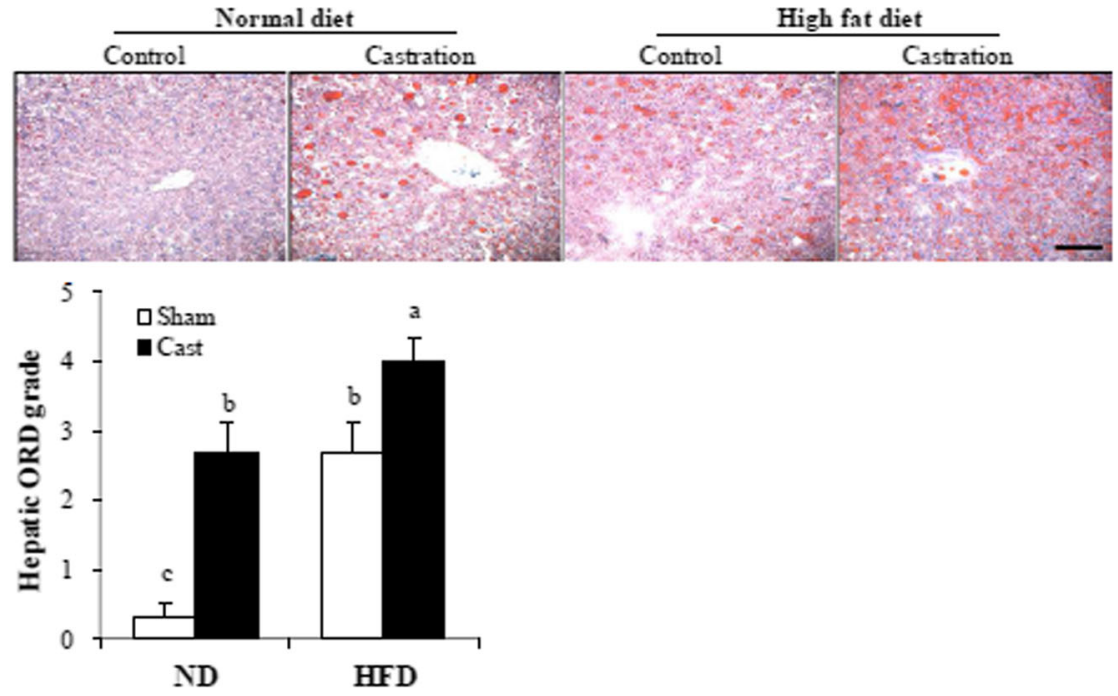

C Lipid contents in muscle

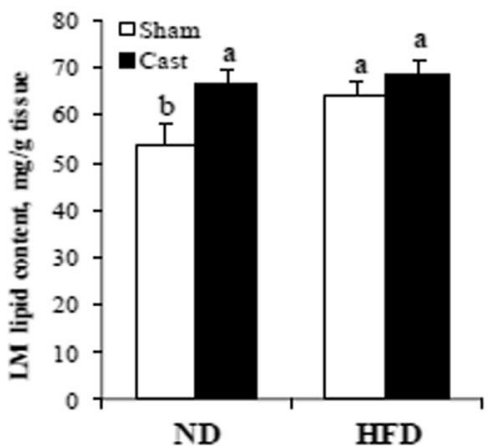

Fig. 1 Body weight (a), Oil red O (ORO) staining in the liver (b), and lipid contents in longissimus dorsi (LD) muscle tissues (c) of sham-operated (Sham) and castrated (Cast) male rats fed an ND or high fat diet (HFD). a Values are means + SE ( $n=7-8$ rats per group). ${ }^{*} P<0.05$ and ${ }^{* *} P<$ 0.01 , significant differences between the Sham and Cast groups. b Scoring of hepatic lipid droplet accumulation $(0=$ none, $5=$ highest). Black bar, $100 \mu \mathrm{m}$. b, c Values are means + SE ( $n=7-8$ per group). ${ }^{a-c}$ Means with different superscripts differ $(P<0.05)$

deficiency and diet type on adiposity. Castration increased the expression of CD36 in the liver and sc and ab fat in the HFD group, but not in the ND group, and in the muscle in the ND group, but not in the HFD group. The activation of CD36 is involved in dietinduced hepatic steatosis $[23,24]$ and is linked to non- 
Table 1 Food intake, food efficiency, and tissue weight of sham-operated (control) and castrated male rats fed either normal diet or high fat diet for 9 weeks

\begin{tabular}{|c|c|c|c|c|c|c|c|}
\hline \multirow{2}{*}{ Items } & \multicolumn{2}{|l|}{ Normal diet } & \multicolumn{2}{|l|}{ High-fat diet } & \multicolumn{3}{|l|}{$P$} \\
\hline & Control & Castration & Control & Castration & Castration & Diet & Interaction \\
\hline Average food intake $1, \mathrm{~g} / \mathrm{d}$ & $20.0 \pm 0.3^{b}$ & $19.2 \pm 0.3^{c}$ & $21.4 \pm 0.4^{a}$ & $19.3 \pm 0.4^{c}$ & $<0.001$ & $<0.001$ & $<0.001$ \\
\hline Initial body weight, $g$ & $254.0 \pm 7.9$ & $242.4 \pm 8.7$ & $257.3 \pm 10.2$ & $247.5 \pm 8.1$ & NS & NS & NS \\
\hline Final body weight at 9 weeks, $g$ & $507.2 \pm 18.4^{\mathrm{a}}$ & $440.9 \pm 17.1^{\mathrm{b}}$ & $507.0 \pm 15.2^{\mathrm{a}}$ & $434.4 \pm 12.3^{b}$ & $<0.001$ & NS & 0.0019 \\
\hline Average food efficiency ${ }^{2}$, gain(g)/intake(g) & $1.6 \pm 0.08^{\mathrm{a}}$ & $1.3 \pm 0.07^{b}$ & $1.7 \pm 0.1^{\mathrm{a}}$ & $1.4 \pm 0.09^{b}$ & $<0.001$ & NS & 0.0033 \\
\hline Abdominal fat, $g$ & $7.3 \pm 0.6^{c}$ & $10.4 \pm 0.7^{b}$ & $10.3 \pm 0.5^{b}$ & $12.59 \pm 0.6^{\mathrm{a}}$ & $<0.001$ & $<0.001$ & NS \\
\hline Subcutaneous fat, $g$ & $18.0 \pm 1.0^{b}$ & $27.2 \pm 2.1^{a}$ & $21.5 \pm 1.5^{b}$ & $31.2 \pm 1.8^{\mathrm{a}}$ & $<0.001$ & 0.04 & NS \\
\hline Total fat ${ }^{3}, \mathrm{~g}$ & $25.3 \pm 1.4^{c}$ & $37.6 \pm 2.5^{b}$ & $31.8 \pm 1.6^{b}$ & $43.8 \pm 2.3^{\mathrm{a}}$ & $<0.001$ & 0.005 & NS \\
\hline Liver, $g$ & $16.2 \pm 1.2^{a}$ & $14.5 \pm 1.5^{\mathrm{ab}}$ & $15.9 \pm 1.1^{a}$ & $11.7 \pm 0.6^{b}$ & 0.02 & NS & NS \\
\hline Longissimus dorsi muscle, g & $10.2 \pm 0.4^{a}$ & $7.9 \pm 0.4^{c}$ & $9.1 \pm 0.3^{b}$ & $7.2 \pm 0.3^{c}$ & $<0.001$ & 0.01 & NS \\
\hline Gastrocnemius, g & $5.5 \pm 0.2^{\mathrm{a}}$ & $5.0 \pm 0.1^{b}$ & $5.0 \pm 0.1^{b}$ & $4.6 \pm 0.1^{b}$ & $<0.001$ & 0.001 & NS \\
\hline Soleus, g & $0.4 \pm 0.04$ & $0.4 \pm 0.06$ & $0.5 \pm 0.06$ & $0.5 \pm 0.07$ & NS & NS & NS \\
\hline
\end{tabular}

Mean \pm SE $(n=7-8)$

NS not significant

${ }^{1}$ Average food intake (g/day): average food intake for whole experimental period ( 9 weeks)

${ }^{2}$ Average food efficiency = average weight gain/food intake ( $g /$ day) for 9 weeks

${ }^{3}$ Total fat $=$ subcutaneous + abdominal fat

${ }^{a-d}$ Means in row with different superscripts differ $(P<0.05)$

alcoholic fatty liver disease in humans [25]. However, activation of $C D 36$ expression in adipose fat cells is unclear. Our findings indicate that HFD-induced upregulation of CD36 expression is associated with increased sc and ab adiposity in addition to hepatic steatosis. Castration increased the expression of $A C C$ and FASN in the liver in the ND group but in muscle in the HFD group. In sc and ab fat, castration increased the expression of $A C C$ and/or FASN in both diet groups; the increased FASN expression in sc fat is consistent with a previous study involving mice [5].

Castration also modulated the expression of genes related to glucose uptake. In sc fat, castration markedly decreased the expression of GLUT4 in the ND and HFD groups. In contrast, castration did not affect the expression of genes related to glucose uptake in the liver or muscle in the ND or HFD group. In ab fat, castration increased the expression of GLUT4 in the HFD, but not in the ND, group. Similarly, Kelley et al. [2] reported that GLUT4 expression was decreased in sc fat but not in ab fat in testicularfeminized compared with normal mice. In this study, the circulating glucose concentration was increased in castrated rats fed an ND but not in those fed an HFD. The increased glucose concentrations may be due to combined effects of the decreased GLUT4 gene expression in sc fat and the decreased muscle mass observed in castrated animals.

Table 2 The serum parameters of sham-operated (control) and castrated-male rats fed either normal diet or high-fat diet at 8-h fasted state

\begin{tabular}{|c|c|c|c|c|c|c|c|}
\hline \multirow[t]{2}{*}{ Item } & \multirow{2}{*}{$\begin{array}{l}\text { Normal diet } \\
\text { Control }\end{array}$} & \multirow[b]{2}{*}{ Castration } & \multicolumn{2}{|l|}{ High-fat diet } & \multicolumn{3}{|l|}{$P$} \\
\hline & & & Control & Castration & Castration & Diet & Interaction \\
\hline Testosterone, pg/mL & $8.30 \pm 1.04^{a}$ & $2.39 \pm 0.13^{b}$ & $11.0 \pm 1.57^{\mathrm{a}}$ & $1.73 \pm 0.39^{b}$ & $<0.001$ & NS & NS \\
\hline Free fatty acid, $\mu \mathrm{Eq} / \mathrm{L}$ & $439 \pm 43.3^{b}$ & $649 \pm 41.3^{\mathrm{a}}$ & $418 \pm 31.7^{b}$ & $533 \pm 25.4^{b}$ & $<0.001$ & NS & NS \\
\hline Insulin, ng/mL & $4.27 \pm 0.38^{\mathrm{a}}$ & $4.25 \pm 0.23^{\mathrm{a}}$ & $2.43 \pm 0.34^{b}$ & $1.38 \pm 0.31^{b}$ & NS & $<0.001$ & NS \\
\hline Total cholesterol, mg/dL & $78.5 \pm 2.87^{b}$ & $105 \pm 3.74^{\mathrm{a}}$ & $97.5 \pm 4.50^{\mathrm{a}}$ & $112 \pm 6.17^{\mathrm{a}}$ & $<0.001$ & 0.02 & NS \\
\hline LDL cholesterol, mg/dL & $7.1 \pm 0.56^{b}$ & $10.2 \pm 1.57^{\mathrm{a}}$ & $15.7 \pm 1.30^{\mathrm{a}}$ & $13.2 \pm 0.69^{\mathrm{a}}$ & NS & $<0.001$ & 0.04 \\
\hline HDL cholesterol, mg/dL & $69.4 \pm 2.76^{b}$ & $95.1 \pm 4.59^{\mathrm{a}}$ & $83.3 \pm 5.03^{\mathrm{a}}$ & $95.7 \pm 5.00^{\mathrm{a}}$ & $<0.001$ & NS & NS \\
\hline Triglyceride mg/dL & $100 \pm 12.3$ & $93.7 \pm 8.30$ & $81.7 \pm 8.35$ & $78.1 \pm 7.24$ & NS & NS & NS \\
\hline Glucose, mg/dL & $179 \pm 17.1^{\mathrm{b}}$ & $237 \pm 10.9^{a}$ & $190 \pm 16.2^{b}$ & $215 \pm 10.1^{\mathrm{ab}}$ & 0.01 & NS & NS \\
\hline
\end{tabular}

Mean \pm SE $(n=7-8)$

NS not significant

${ }^{a-b}$ Means in row with different superscripts differ $(P<0.05)$ 


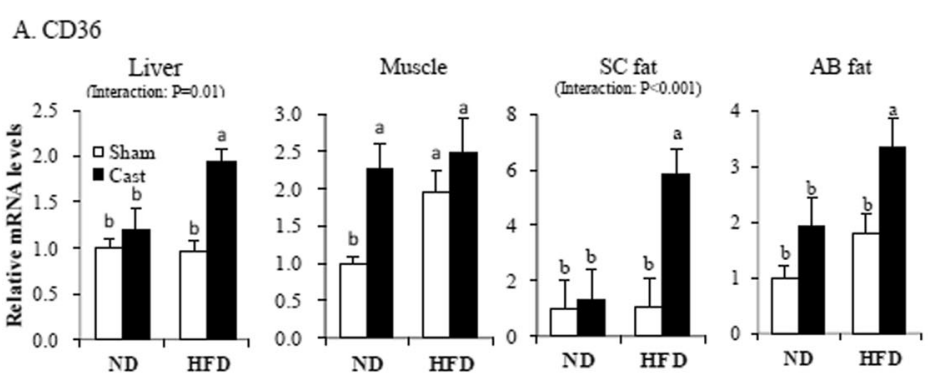

\section{B. ACC and FASN}

$\mathrm{ACC}$
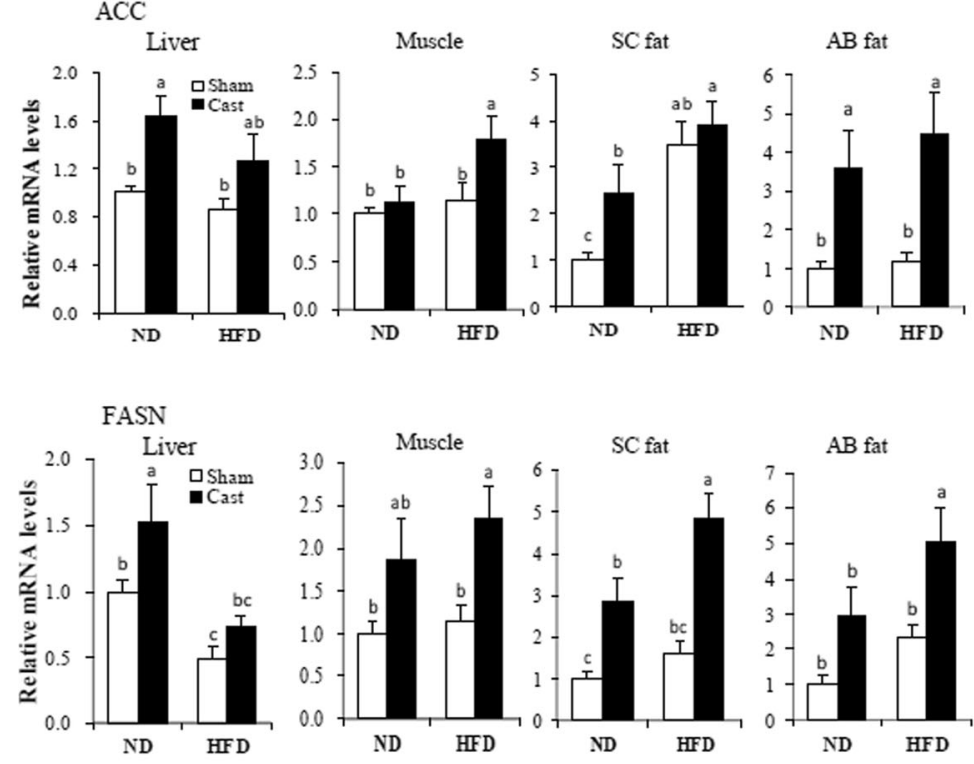

C. GLUT 2 and GLUT 4

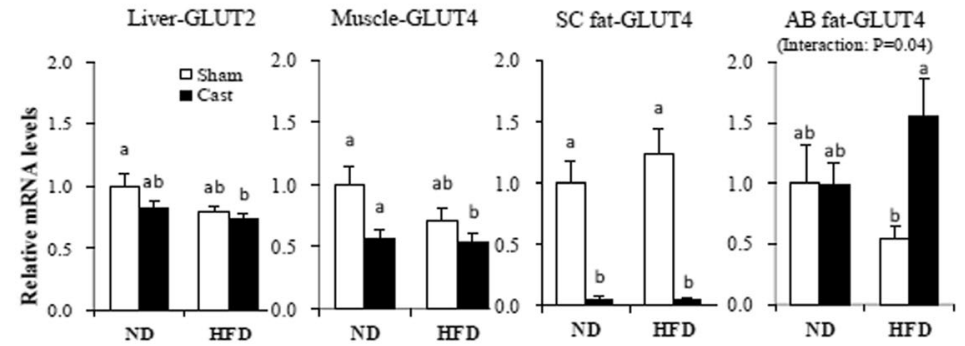

D. Cholesterol uptake and androgen signaling genes

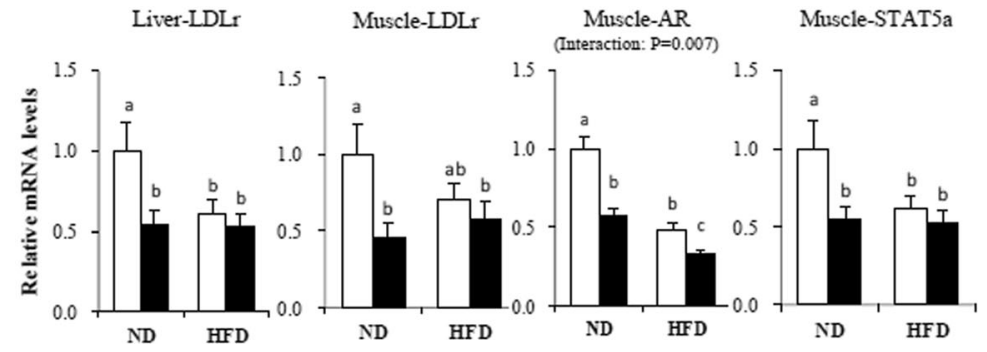

Fig. 2 (See legend on next page.) 
(See figure on previous page.)

Fig. 2 Expression levels of genes related to FA uptake (a), FA synthesis (b), glucose transporter (c), and cholesterol uptake and androgen signaling (d) in the liver, muscle, subcutaneous fat (sc), and abdominal fat (ab) of sham-operated (Sham) and castrated (Cast) male rats fed a normal diet (ND) or high fat diet (HFD). mRNA levels were determined by real-time PCR, and the values of sham-operated rats fed the ND were normalized to 1.0. Values are means + SE $(n=7-8) .{ }^{a-c}$ Means with different superscripts differ $(P<0.05)$. CD36 cluster of differentiation $36, A C C$ acetyl-coA carboxylase, FASN fatty acid synthase, GLUT2 glucose transporter 2, GLUT4 glucose transporter 4, LDLr low-density lipoprotein receptor, AR androgen receptor, STAT5a signal transducer and activator of transcription 5a

Collectively, our results suggest that testosterone deficiency regulates adiposity in a tissue-specific and dietdependent manner (Supporting Fig. S2). Upregulation of $A C C$ and FASN expression may have contributed to the initial increase in hepatic adiposity in rats fed an ND, and activation of CD36 expression was responsible for the development of hepatic steatosis in rats fed an HFD. The increased adiposity in the muscle tissues of rats in the ND group may be due to FA uptake because the expression of CD36, but not that of $A C C, F A S N$, or GLUT4, was increased. In sc fat, the expression of genes related to FA synthesis was upregulated and that of genes related to glucose transporter was downregulated in the ND and HFD groups. Glucose is a major substrate for FA synthesis in fat cells [26]. Thus, further clarification of the contribution of FA synthesis to the castration-induced increase in sc fat accumulation is needed. In $a b$ fat, upregulation of genes related to FA synthesis may have contributed to the ND-induced increase in adiposity. Moreover, an HFD may aggravate ab adiposity by activating the expression of genes related to FA uptake (CD36) and synthesis, as GLUT4 expression was increased in the HFD group but unaffected in the ND group. In other words, in ab fat, a portion of glucose precursors for FA synthesis may be derived from the castration-induced increase in circulating glucose concentration. Overall, our results suggest that a normal circulating testosterone level is important for regulating FA uptake and synthesis in all tissues and that testosterone deficiency differentially deregulates lipid metabolism. This may result in elevation of the circulating free FA and cholesterol concentrations, leading to adiposity in the liver, muscle, and ab and sc fat depots.

Our results also suggest that a low testosterone level is associated with an increased risk of type- 2 diabetes, in that castrated rats exhibited reduced expression of genes related to glucose uptake in sc fat as well as an elevated circulating glucose concentration. Following castration, we found increased blood cholesterol levels, which may be caused by decreased cholesterol uptake in liver and muscle, as the expression of $L D L r$ in the liver and muscle was decreased by castration of the rats.

The increased adiposity of the liver and fat tissues in the castrated rats may have been differentially induced in the ND and HFD groups. The ND contained more carbohydrate $(64 \%)$ and less fat (7\%) than the HFD $(41 \%$ carbohydrate and $24 \%$ fat). The higher insulin concentrations observed in rats fed an ND, compared with those fed an HFD, may reflect higher carbohydrate consumption. In the ND group, the castrated rats had higher glucose concentrations than non-castrated animals; the elevated glucose concentrations in the castrated rats may partially contribute to the increased adiposity in liver and fat tissues. In the ND group, the increased $A C C$ and FASN expression levels in the liver and $\mathrm{Sc}$ and $\mathrm{ab}$ fat of the castrated rats, compared with non-castrated rats, support our assumption that high carbohydrate consumption causes elevated adiposity in these tissues, although there is a need to consider the reduced GLUT4 expression in the sc fat of the castrated rats, compared with non-castrated rats. Meanwhile, the rats fed an HFD had higher total cholesterol concentrations than rats fed an ND. The elevated total cholesterol concentrations in the castrated rats fed an HFD may partially contribute to the increased adiposity in the liver and in $\mathrm{ab}$ and sc fats. In the HFD group, the increased CD36 expression in the liver and $a b$ and sc fat of the castrated rats, compared with non-castrated rats, may partially support the implication that high fat consumption causes the increased adiposity in these tissues.

\section{Testosterone deficiency decreases body and muscle weights and downregulates androgen signaling}

We found that castration decreased the body, longissimus dorsi, and gastrocnemius weights of the rats. Castration also decreased $S T A T 5 a$ and $A R$ expression in the longissimus dorsi muscle, and $A R$ expression was further decreased by the HFD. Thus, castration-induced testosterone deficiency deregulates AR signaling, and a normal testosterone level is required for AR signaling. Furthermore, the HFD augmented the castration-induced decrease in muscle mass and $A R$ expression. Therefore, proper nutrition is important for maintaining muscle mass in the presence of a low testosterone level because a low testosterone level may aggravate the HFD-induced decrease in muscle mass. Decreased muscle mass may decrease glucose utilization by muscles, leading to hyperglycemia and deregulation of glucose homeostasis. This may in part explain the increased ab adiposity under testosterone deficiency in the ND and HFD groups. 
Zirkin and Tenover [27] reported that the circulating testosterone level typically declines with age. Indeed, treatment of older men with growth hormone and testosterone improved their lean body mass [28]. Testosterone deficiency, which can be caused by hypogonadism, central obesity, or androgen-deprivation in prostate cancer patients, is related with metabolic syndromes including insulin resistance and type-2 diabetes [29]. In animals, an HFD differentially influences body metabolism and metabolic disease with age. HFD feeding resulted in greater increases in body weight and serum total cholesterol and glucose levels in older mice compared with younger mice [30]. Our study suggests that consumption of an HFD by elderly men, who generally have low testosterone levels, may promote the development of metabolic syndrome.

In this study, the rats were castrated at 6 weeks of age, when they were immature. Consequently, castration may have affected the overall development of the animals and contributed to physiological differences relative to noncastrated animals. In men, testosterone levels typically decline at old age. Therefore, there may be a limitation for direct implication of our findings to men, since rats were castrated at immature age.

A testosterone supplementation study to the castrated rats could validate the testosterone deficiency model by castration, although we did not conduct the experiment.

\section{Conclusions}

In conclusion, castration-induced testosterone deficiency influenced body adiposity and the expression of genes related to lipid metabolism and glucose uptake in a tissuespecific and diet-dependent manner in rats (Supporting Fig. S2). Testosterone deficiency initially induced hepatic fat accumulation by activating the expression of genes related to FA synthesis; the HFD increased hepatic fat accumulation further by upregulating genes related to FA uptake. In muscle, testosterone deficiency in rats fed the ND, but not in those fed the HFD, induced fat accumulation by activating CD36 expression. In sc fat, contribution of glucose for FA synthesis is questionable for the castration-induced increase in adiposity because castration decreased glucose transporter expression in both diet groups although it increased FA synthesis gene expression. Upregulation of genes related to FA uptake may be involved in the HFDinduced increase in sc fat. The ab fat accumulation of castrated rats fed a ND may be increased via upregulation of genes related to FA synthesis and that fed an HFD was further induced via upregulation of genes related to FA uptake, glucose transporter, and FA synthesis. The castrationinduced downregulation of genes related to glucose transporter in sc fat and increase in the circulating glucose concentration imply the involvement of testosterone deficiency in the pathogenesis of type- 2 diabetes.

\section{Supplementary information}

Supplementary information accompanies this paper at https://doi.org/10. 1186/s12263-020-00673-1.

Additional file 1: Table S1. Composition of the experimental diets ( $\mathrm{g}$ / kg). Table S2. Composition of mineral mix in experimental diets. Table S3. Composition of vitamin mix in experimental diets. Table 4. Primers sequences used in real-time PCR. Figure S1. Summary of castration effects on adiposity (TG accumulation), blood parameters, and gene expression under normal diet (ND: A) and high-fat diet (HFD) feeding (B) in liver, longissimus dorsi (LD) muscle, and subcutaneous (sc) and abdominal (ab) fats of male rats. Figure S2. Summary of castration effects on adiposity (TG accumulation), blood parameters, and gene expression under normal diet (ND: A) and high-fat diet (HFD) feeding (B) in liver, longissimus dorsi (LD) muscle, and subcutaneous (sc) and abdominal (ab) fats of male rats. The arrow indicates changes (up or down) of parameters by castration. $T$ testosterone, TG triglycerol, FFA free fatty acid, CD36 cluster of differentiation 36, ACC acetyl-CoA carboxylase, FASN fatty acid synthase, GLUT2 glucose transporter 2, GLUT4 glucose transporter 4, LDLr low density lipoprotein receptor, AR androgen receptor, STAT5 a signal transducer and activator of transcription $5 \mathrm{a}$. ND = no difference.

\section{Abbreviations}

ACC: Acetyl-CoA carboxylase; AR: Androgen receptor; CD36: Fatty acid translocase; FA: Fatty acid; FASN: Fatty acid synthase; GLUT2: Glucose transporter 2; GLUT4: Glucose transporter 4; HFD: High-fat diet; LDLr: Lowdensity lipoprotein receptor; ND: Normal diet

\section{Acknowledgements}

We would like to thank all the participants in the trial.

\section{Authors' contributions}

MB and JYJ designed and planned the study. JYJ, SJP, SPY, JOL, and JSL carried out the feeding trial, laboratory analysis, and statistical analysis. MB, JYJ, SJP, JOL, JSL, MNH, and HJL wrote the manuscript. All authors read and approved the final manuscript.

\section{Funding}

This study was supported by the Korea Research Fellowship Program through the National Research Foundation of Korea (NRF) funded by the Ministry of Science and ICT (2019H1D3A1A01102738).

\section{Availability of the data and materials}

Not applicable

\section{Ethics approval and consent to participate}

All experimental procedures involving animals were approved by the Chonnam National University (CNU) Institutional Animal Use and Care Committee (permission number CNU IACUC-YB-R-2010-13). All animal management procedures followed the standard operating protocols of CNU.

\section{Consent for publication}

Not applicable

\section{Competing interests}

The authors declare no competing interests.

\section{Author details}

${ }^{1}$ Department of Agricultural Biotechnology, College of Agriculture and Life Sciences, Seoul National University, 1 Gwanak-ro, Gwanak-gu, Seoul 08826, Republic of Korea. ${ }^{2}$ Research Institute of Agriculture and Life Sciences, College of Agriculture and Life Sciences, Seoul National University, Seoul, Republic of Korea. ${ }^{3}$ Institutes of Green Bio Science Technology, Pyeongchang-daero, Daehwa-myeon, Pyoengchang-gun, Gangwon-do 25354, Republic of Korea. ${ }^{4}$ National Institute of Animal Science, Rural Development Administration, Wanju-gun, Jeollabuk-do 55365, Republic of Korea. 
Received: 29 February 2020 Accepted: 5 August 2020

Published online: 17 August 2020

\section{References}

1. Kelly DM, Jones TH. Testosterone: a metabolic hormone in health and disease. J Endocrinol. 2013;217:R25-45.

2. Kelly DM, Akhtar S, Sellers DJ, Muraleedharan V, Channer KS, Jones TH. Testosterone differentially regulates targets of lipid and glucose metabolism in liver, muscle and adipose tissues of the testicular feminised mouse. Endocrine. 2016;54:504-15.

3. Mårin P, Lönn L, Andersson B, Odén B, Olbe L, Bengtsson BA, et al. Assimilation of triglycerides in subcutaneous and intraabdominal adipose tissues in vivo in men: effects of testosterone. J Clin Endocrinol Metab. 1996; 81:1018-22.

4. Baik M, Nam YS, Piao MY, Kang HJ, Park SJ, Lee JH. Liver-specific deletion of the signal transducer and activator of transcription 5 gene aggravates fatty liver in response to a high-fat diet in mice. J Nutr Biochem. 2016;29:56-63.

5. Dubois V, Laurent MR, Jardi F, Antonio L, Lemaire K, Goyvaerts L, et al. Androgen deficiency exacerbates high-fat diet-induced metabolic alterations in male mice. Endocrinology. 2016;157:648-65

6. Allan CA, Strauss BJ, Burger HG, Forbes EA, McLachlan RI. Testosterone therapy prevents gain in visceral adipose tissue and loss of skeletal muscle in nonobese aging men. J Clin Endocrinol Metab. 2008;93:139-46.

7. Jones TH. Testosterone deficiency: a risk factor for cardiovascular disease? Trends Endocrinol Metab. 2010;21:496-503.

8. Nikolaenko L, Jia Y, Wang C, Diaz-Arjonilla M, Yee JK, French SW, et al. Testosterone replacement ameliorates nonalcoholic fatty liver disease in castrated male rats. Endocrinology. 2014;155:417-28.

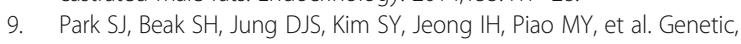
management, and nutritional factors affecting intramuscular fat deposition in beef cattle - a review. Asian-Australas J Anim Sci. 2018;31:1043-61.

10. Frühbeck G, Méndez-Giménez L, Fernández-Formoso JA, Fernández S, Rodríguez A. Regulation of adipocyte lipolysis. Nutr Res Rev. 2014;27:63-93.

11. Jeong J, Kwon EG, Im SK, Seo KS, Baik M. Expression of fat deposition and fat removal genes is associated with intramuscular fat content in longissimus dorsi muscle of Korean cattle steers. J Anim Sci. 2012;90:204453.

12. Zhou J, Febbraio M, Wada T, Zhai Y, Kuruba R, He J, et al. Hepatic fatty acid transporter Cd36 is a common target of LXR, PXR, and PPARgamma in promoting steatosis. Gastroenterology. 2008;134:556-67.

13. Tajar A, Huhtaniemi IT, O'Neill TW, Finn JD, Pye SR, Lee DM, et al. Characteristics of androgen deficiency in late-onset hypogonadism: results from the European Male Aging Study (EMAS). J Clin Endocrinol Metab. 2012;97:1508-16.

14. Herbst KL, Bhasin S. Testosterone action on skeletal muscle. Curr Opin Clin Nutr Metab Care. 2004;7:271-7.

15. Baik M, Yu JH, Hennighausen L. Growth hormone-STAT5 regulation of growth, hepatocellular carcinoma, and liver metabolism. Ann N Y Acad Sci. 2011;1229:29-37.

16. Winzell MS, Ahren B. The high-fat diet-fed mouse: a model for studying mechanisms and treatment of impaired glucose tolerance and type 2 diabetes. Diabetes. 2004;53(Suppl 3):S215-9.

17. Costa RR, Villela NR, Md S, Boa BC, Cyrino FZ, Silva SV, et al. High fat diet induces central obesity, insulin resistance and microvascular dysfunction in hamsters. Microvasc Res. 2011:82:416-22.

18. Alberti KG, Zimmet P, Shaw J. Metabolic syndrome-a new worldwide definition. A consensus statement from the International Diabetes Federation. Diabet Med. 2006;23:469-80.

19. Kwon DH, Kang W, Nam YS, Lee MS, Lee IY, Kim HJ, et al. Dietary protein restriction induces steatohepatitis and alters leptin/signal transducers and activators of transcription 3 signaling in lactating rats. J Nutr Biochem. 2012; 23:791-9.

20. Baik M, Lee MS, Kang HJ, Park SJ, Piao MY, Nguyen TH, et al. Muscle-specific deletion of signal transducer and activator of transcription 5 augments lipid accumulation in skeletal muscle and liver of mice in response to high-fat diet. Eur J Nutr. 2017:56:569-79.

21. Folch J, Lees M, Sloane Stanley GH. A simple method for the isolation and purification of total lipides from animal tissues. J Biol Chem. 1957;226:497509.
22. Kim Y, Iwashita S, Tamura T, Tokuyama K, Suzuki M. Effect of high-fat diet on the gene expression of pancreatic GLUT2 and glucokinase in rats. Biochem Biophys Res Commun. 1995;208:1092-8.

23. Inoue M, Ohtake T, Motomura W, Takahashi N, Hosoki Y, Miyoshi S, et al. Increased expression of PPARgamma in high fat diet-induced liver steatosis in mice. Biochem Biophys Res Commun. 2005;336:215-22.

24. Koonen DPY, Jacobs RL, Febbraio M, Young ME, Soltys CLM, Ong H, et al. Increased hepatic CD36 expression contributes to dyslipidemia associated with diet-induced obesity. Diabetes. 2007;56:2863-71.

25. Miquilena-Colina ME, Lima-Cabello E, Sanchez-Campos S, Garcia-Mediavilla MV, Fernandez-Bermejo M, Lozano-Rodriguez T, et al. Hepatic fatty acid translocase CD36 upregulation is associated with insulin resistance, hyperinsulinaemia and increased steatosis in non-alcoholic steatohepatitis and chronic hepatitis C. Gut. 2011;60:1394-402.

26. Saltiel AR, Kahn CR. Insulin signalling and the regulation of glucose and lipid metabolism. Nature. 2001;414:799-806.

27. Zirkin BR, Tenover JL. Aging and declining testosterone: past, present, and hopes for the future. J Androl. 2012;33:1111-8.

28. Sattler FR, Castaneda-Sceppa C, Binder EF, Schroeder ET, Wang Y, Bhasin S, et al. Testosterone and growth hormone improve body composition and muscle performance in older men. J Clin Endocrinol Metab. 2009;94:19912001.

29. Bain J. Testosterone and the aging male: to treat or not to treat? Maturitas. 2010;66:16-22.

30. Korou LM, Doulamis IP, Tzanetakou IP, Mikhailidis DP, Perrea DN. The effect of biological age on the metabolic responsiveness of mice fed a high-fat diet. Lab Anim. 2013;47:241-4.

\section{Publisher's Note}

Springer Nature remains neutral with regard to jurisdictional claims in published maps and institutional affiliations.
Ready to submit your research? Choose BMC and benefit from:

- fast, convenient online submission

- thorough peer review by experienced researchers in your field

- rapid publication on acceptance

- support for research data, including large and complex data types

- gold Open Access which fosters wider collaboration and increased citations

- maximum visibility for your research: over $100 \mathrm{M}$ website views per year

At $\mathrm{BMC}$, research is always in progress.

Learn more biomedcentral.com/submissions 